\title{
WMW-Themenheft „Kardiologische Rehabilitation“
}

\author{
Christiane Marko
}

Online publiziert: 9. November 2017

(c) Springer-Verlag GmbH Austria 2017

\section{Sehr geehrte KollegInnen!}

Die Kardiologische Rehabilitation stellt einen wesentlichen Bestandteil der Versorgung von Herz-Kreislaufpatienten dar. Es ist ein multidisziplinäres Therapiekonzept, das an die individuellen Bedürfnisse des Patienten angepasst wird, wobei die tragenden Säulen die medizinische Trainingstherapie, die psychosoziale Beratung, die Ernährungsmedizin und krankheitsbezogene Schulungen ausmachen.

Metaanalysen von internationalen Studien bewiesen eine Verbesserung der Lebensqualität und eine Senkung der Morbidität und Mortalität. Weitere Ziele sind auch die bestmögliche Wiedereingliederung in das soziale und berufliche Umfeld.

In den Präventionsleitlinien der ESC 2016 wurde die kardiologische Rehabilitation nach einem akuten Koronarsyndrom, bei Herzinsuffizienz und nach Revaskularisation mit der höchsten Empfehlungsklasse und dem höchsten Evidenzgrad (Klasse 1A) eingestuft.

Trotzdem nehmen immer noch viel zu wenig HerzKreislaufpatienten überhaupt und insbesondere Frauen an kardiologischen Rehabilitationsprogrammen teil.

Dieses Themenheft soll, neben den Leitlinien und der Situation in Österreich und in Europa, auch auf die Vielfältigkeit der Aufgaben, die das Rehabilitationsteam zu bewältigen hat, aufmerksam machen.
Den Anfang macht ein Beitrag von Doz. Dr. Thomas Berger über die Neuigkeiten in den Präventionsleitlinien der ESC 2016, gefolgt von Prim. Doz. Dr. Berent, der die Therapieoptionen von Fettstoffwechselstörungen zusammenfasst und Prim. Doz. Dr. Alber, der sich der Antiplättchentherapie widmet. Dies sind zwei Themenkreise mit vielen medizinischen Neuigkeiten in den letzten Jahren, die sehr personalisiert vom Rehabilitationsteam umgesetzt werden. Prim. Doz. Dr. Altenberger schreibt über die Rehabilitation von Herzinsuffizienzpatienten, wobei auch hier die aktuellen ESC-Guidelines einfließen.

Nicht zu unterschätzen für die Prognose und Lebensqualität sind psychokardiologische Aspekte, diese werden von Frau Mag. Dr. Fuchs-Stritzek erläutert. Den Abschluss bildet jeweils ein Artikel über die Situation der kardiologischen Rehabilitation in Europa von Prim. Doz. Dr. Benzer und einer über die in Österreich von Prim. Prof. Dr. Niebauer.

Ich hoffe, dass die Lektüre dieses Themenhefts alle LeserInnen überzeugen wird, dass ein Rehabilitationsprogramm nach einem akuten kardialen Ereignis einen wichtigen Stellenwert einnimmt und unbedingt dem Patienten zu empfehlen ist.

\section{Prim. Dr. Christiane Marko}

Interessenkonflikt C. Marko gibt an, dass kein Interessenkonflikt besteht. 